\title{
Immunocytochemical strategies for signal enhancement in post-embedding immunogold procedure
}

\section{G. Grondin}

Microscopy Service, Department of Biology, Faculty of Sciences, University of Sherbrooke, Sherbrooke, Québec, Canada, J1K-2R1

Post-embedding immunogold cytochemistry is the method of choice for high-resolution localisation of secretory antigens (1-3). However with this approach, labelling is restricted to those epitopes that are expressed at the surface of the section. Because these constitute a minor fraction of the epitopes, the sensitivity becomes a critical factor. Here we report the result of our efforts to increase the sensitivity of post-embedding immunogold labelling.

Immediately after sacrifice, pieces of tissue $\left(1 \mathrm{~mm}^{3}\right)$ of rat pancreas were fixed at room temperature for $120 \mathrm{~min}$. in $2 \%$ paraformaldehyde, $0.25 \%$ glutaraldehyde in $100 \mathrm{mM}$ cacodylate buffer $\mathrm{pH} 7.4$ with $4 \%$ sucrose. After washing in the same buffer, samples were post-fixed overnight at $4^{\circ} \mathrm{C}$ in $2 \%$ paraformaldehyde in the same buffer. After progressive dehydration in ethanol they were passed through propylene oxide and embedded in Embed 812 . Thin sections $(70 \mathrm{~nm})$ were mounted on uncoated 200 mesh hexagonal nickel grids in order to have both faces of the section exposed and were processed according to our modified immunogold protocol. The following modifications were found to enhance sensitivity; $1^{0}$, immersion of grid in each step of incubation for labelling the epitopes on each side of the section; $2^{0}$, two short incubations with the primary antibody with many washing between incubations to exploit the high affinity and high avidity antibodies present in the serum.

Here we describe in detail our incubation protocol: $1^{0}$ - Distilled water $3 \times 2$ min.; $2^{0}$ - PBST 1 x 5 min. (phosphate buffer saline with $0.1 \%$ of Tween 20 ) $; 3^{0}$ - PBSTC 1 x 15 min. (PBST with $2 \%$ of albumin chicken fraction V \#3014 Sigma); 40- Ac-alpha-Amylase 1 x 40 min.(a well characterised antiserum (1-3) at a dilution of 1:200 in PBSTC; $5^{0}$ - PBST 3 x 5 min.; $6^{0}-$ Acalpha-Amylase in PBSTC 1:200, 1 x 40 min.; $7^{0}$ - PBST 3 x 10 min.; $8^{0}-1$ x 30 min. in $10 \mathrm{~nm}$ proteinA-gold complex dilution O.D.520nm $=0.1$ in PBSTPEG (PBST with $0.05 \%$ of polyethylene glycol 20,000 and $0.2 \%$ of albumin chicken fraction V); $9^{0}$ - PBST $3 \times 10$ min.; $10^{\circ}$ - Distilled water $3 \times 10 \mathrm{~min}$. The sections were stained with uranyl acetate and lead citrate before examination with the electron microscope.

One can appreciate in Figure 1, the labelling in the rough endoplasmic reticulum (arrows) with an absence of labelling in the nucleus $(\mathrm{Nu})$ and the mitochondria (Mi). Notice in Figure 2, the quality of the ultrastructure, more particulary the Golgi apparatus (Go) together with the signal intensity in Golgi and Zymogen granules $(\mathrm{Zg})$ whereas the mitochondria are devoid of any labelling. Figure 3 is a high magnification of Zymogen granule to demonstrate the high number of epitopes labelled with gold particles. Figure 4 is a control with an unrelated antibody (Acinsulin 1:200) that illustrates the low level of non-specific labelling (arrows). This new fixation protocol together with the simple modification of labelling conditions were found to enhance sensitivity of post-embedding immunogold localisation.

1-Grondin et al.Eur.J.Cell.Biol. 57 (1992) 155-164.

2-Grondin et al.J.Histochem.Cytochem. 44 (1996) 357-368.

3-Grondin et al.,J.Histochem.Cytochem. 47 (1999) 489-497. 

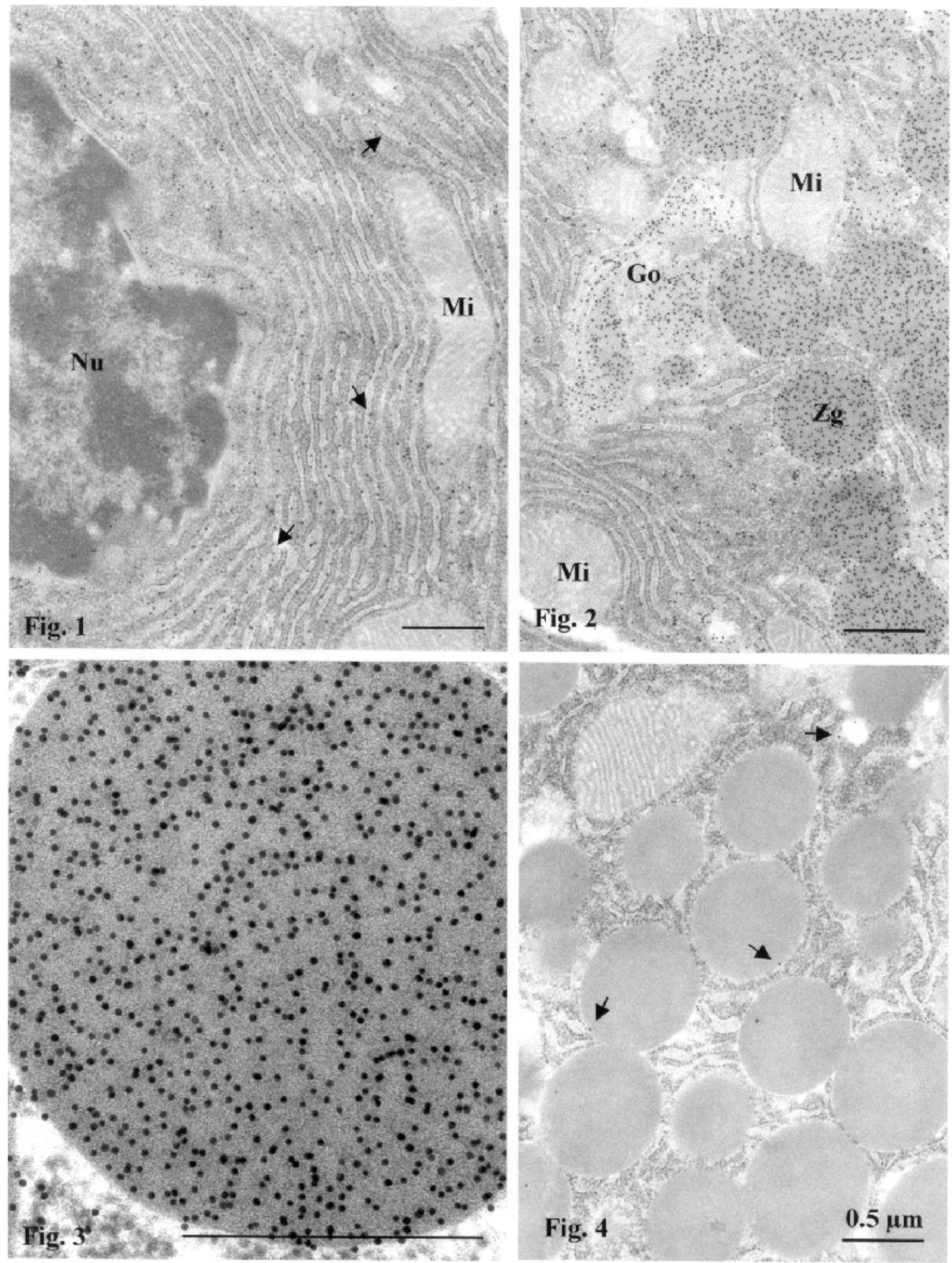